\title{
Exercise training on chronotropic response and exercise capacity in patients with type 2 diabetes mellitus
}

\author{
LI JIN $^{1-3 *}$, GAO MIN ${ }^{1-3 *}$, CHEN WEI $^{1-3}$, HE MIN $^{2}$ and ZHOU JIE $^{3}$ \\ ${ }^{1}$ Department of Rehabilitation Medicine, Center Hospital of Xuzhou, Xuzhou, Jiangsu 221009; ${ }^{2}$ Department of \\ Cardiopulmonary Rehabilitation, Rehabilitation Hospital of Xuzhou, Xuzhou, Jiangsu 221003; ${ }^{3}$ Academy of \\ Medical Technology, Medical College of Xuzhou, Xuzhou, Jiangsu 221004, P.R. China
}

Received June 29, 2016; Accepted November 30, 2016

DOI: $10.3892 /$ etm.2017.4084

\begin{abstract}
The study was designed to observe the effects and relationship of exercise on chronotropic response (CR) and exercise capacity in patients with type 2 diabetes mellitus (T2DM). A total of 30 patients with T2DM underwent symptom-limited cardiopulmonary exercise testing (CPET) after excluding contraindication. For each subject individualized exercise prescription was formulated, and they received 12 weeks of exercise training after CPET retest to complete the comparison of CR indicators, including the ratio of maximum exercise heart rate to predicted maximum heart rate value (rHR), heart rate reserve rate (HRRes), heart rate recovery (HRR) of 1-6 min after exercise termination $\left(\mathrm{HRR}_{1-6}\right.$ ), exercise capacity (peak $\mathrm{VO}_{2} / \mathrm{kg}$ ) and other indicators. The results showed that after 12 weeks of exercise treatment, $\mathrm{rHR}, \mathrm{HRR} e s, \mathrm{HRR}_{1-6}$, and peak $\mathrm{VO}_{2} / \mathrm{kg}$ were significantly higher than before $(\mathrm{P}<0.05)$, with peak $\mathrm{VO}_{2} / \mathrm{kg}$ being positively correlated to $\mathrm{rHR}$ and HRRes $(\mathrm{P}<0.01)$. In conclusion, exercise training can improve cardiac dysfunction, abnormal HRR, enhance exercise capacity and adaptability of the cardiovascular system to exercise stress in T2DM patients.
\end{abstract}

\section{Introduction}

Diabetes mellitus causes significant changes in socioeconomic life and its prevalence is on the increase every year. China has become the country with the highest number of diabetic cases (1). Several studies have shown that regular exercise can improve glycemic control and increase insulin sensitivity in the patients with diabetes mellitus (DM), along with reduction in body weight, control blood lipid and blood pressure and risk

Correspondence to: Dr Chen Wei, Department of Rehabilitation Medicine, Center Hospital of Xuzhou, 57 Heping Road, Xuzhou, Jiangsu 221009, P.R. China

E-mail: chen_wei1212@163.com

*Contributed equally

Key words: type 2 diabetes mellitus, exercise training, chronotropic response, heart rate recovery, exercise capacity of macrovascular and microvascular complications (2). Out of the different significant treatment methods, exercise has been given increased attention (3).

Chronotropic response (CR) defined as the function of the heart to increase its rate commensurate with the rise of the body's metabolic demand during exercise. Not reaching a certain extent is termed chronotropic incompetence (CI), which is also known as an independent predictor of incidence of severe cardiovascular events, and the all-cause mortality (4). It has been indicated that CI was closely related to the impairment of cardiac autonomic nervous function (5). Cardiac autonomic nerves refer to the sympathetic and parasympathetic nerves that administer circulation system including heart, lung and peripheral blood vessels. Due to physiological or pathological factors, the balance of coordination and confrontation is changed, which is described as cardiovascular autonomic neuropathy (CAN) (6). It has been found that $\mathrm{CAN}$, which can cause painless myocardial infarction and sudden death, is one of the important reasons for the increased mortality of type 2 DM (T2DM) patients, even at the initial stage of T2DM (7).

In the present study, heart rate reserve rate (HRRes) and heart rate value (rHR) were selected as the evaluation criteria (4): i) rHR: The ratio of maximum heart rate in exercise to predict maximum heart rate, $<85 \%$ was abnormal; and ii) HRRes: Heart rate reserve rate, $<80 \%$ was abnormal.

Heart rate recovery (HRR) refers to the difference of peak heart rate (HRpeak) in exercise testing and heart rate during convalescence after exercise ceased, is one of the common indicators for assessing cardiac autonomic nerve function, thus some scholars regarded it as the other evaluating indicator of CR (8). In the past, the studies of HRR mainly focused on coronary heart disease, heart failure and other fields (9), minority of research was on T2DM patients. Vagus nerve lesions mainly occur in the early stage in T2DM patients, while abnormal HRR after exercise was directly related to vagal nerve activity (4), which was a sign of reduced vagal activity (10) also as independent predictor of incidence of future cardiovascular events and mortality in T2DM patients (11). While there are various standards of cut-off point for assessing abnormal HRR, the most generally accepted and employed by specialists worldwide (4) was slow walking after exercise testing, $1 \mathrm{~min}$ of HRR of $<18$ beats/min (bmp) or $2 \mathrm{~min}$ of recovery 
of $<42$ bmp. If special circumstances can be discovered, observation time could be extended until abnormal manifestations or symptoms have disappeared. For general observation for 6 min after test termination, this study may record HRR of 1-6 min after movement as $\mathrm{HRR}_{1-6}$.

Exercise capacity directly reflects the level of individual health status and self-care ability. During exercise, the oxygen requirement of the body increases while the oxygen uptake of the cells increases correspondingly. When the amount of exercise raises to a certain degree, cell uptake of oxygen will reach a plateau with no further increase i.e., to achieve the maximal oxygen uptake (peak $\mathrm{VO}_{2}$ ), which reflects the human maximum aerobic capacity and cardiopulmonary reserve capacity. However, due to differences in body weight, peak $\mathrm{VO}_{2}$ also has difference even if completed with the same power load. So this study used peak $\mathrm{VO}_{2} / \mathrm{kg}$ to measure the exercise ability of an individual (12).

A previous study on exercise therapy focused on the control of blood glucose, blood pressure and the improvement of metabolic parameters and body composition (13). However, to the best of our knowledge, few studies have shown the effects of exercise therapy on CR, HRR and exercise capacity in patients with T2DM. In the present study, CR, HRR $\mathrm{H}_{1-6}$ and the exercise capacity of T2DM patients were determined by symptom-limited cardiopulmonary exercise testing (CPET). The patients were given a 12-week exercise therapy according to individual exercise prescription as formulated. The impact of exercise training on the improvement of CI, and regulation of the function of cardiac autonomic nerves and exercise capability were investigated, exploring the possible mechanism, in order to find new evidence of the mediation of individual exercise treatment on CR, HRR of T2DM patients.

\section{Patients and methods}

Patients observation. Of the patients receiving cardiopulmonary rehabilitation exercise therapy in the Center Hospital and Rehabilitation Hospital of Xuzhou from October 2014 to October 2015, 30 cases were diagnosed with T2DM. The average age was $59.43 \pm 7.81$ years, with 17 males and 13 females. There were 10 cases with coronary heart disease and 7 cases with hypertension. All of the patients were treated conventionally as before.

Inclusion criteria were as follows: Patients with a clear history of T2DM, the diagnostic criteria of T2D references to the Standards of Medical Care in Diabetes - 2010 of ADA (14); age 40-75 years; middle school education level or higher; to be conscious and stable to cooperate with the inspection; patients with no regular exercise history; no use of $\beta$-blockers, atropine, nitrates and antiarrhythmic drugs in $48 \mathrm{~h}$. All the patients signed informed consent.

Exclusion criteria were, patients with psychosis and dyscinesia; patients with serious heart, brain, kidney disease not yet stabilized; patients with non-sinus rhythm, atrial fibrillation, atrial flutter, the conduction block and ventricular rhythmia patients; cases with infection, acute metabolic disorders, diabetic foot, diabetic retinopathy and other serious complications of DM; patients with systolic blood pressure (SBP) 1,200 $\mathrm{mmHg}$ and/or diastolic blood pressure $(\mathrm{DBP}) \geq \geq \operatorname{Pod} \operatorname{pr}(15)(1 \mathrm{mmHg}=0.133 \mathrm{kPa})$, blood glucose before exercising, diab/l or $\mathrm{Hg}=0.1 / 1$ (15); and poor adherence to exercise therapy.

Methods. All subjects underwent symptom-limited CPET, with K4b2 exercise cardiopulmonary function test and metabolic analysis system from Cosmed (Rome, Italy) and CASE treadmill exercise machine from GE Healthcare (Piscataway, NJ, USA). To formulate individualized exercise prescription on the basis of test results and to require a 12-week exercise training, equipment from Welch Allyn Inc. (Skaneateles Falls, NY, USA), telemetric electrocardiogram (ECG) monitor, finger pulse oximeter and related fitness equipment from our department was used. The repeated measure by symptomlimited CPET was undertaken 12 weeks later.

All subjects received symptom-limited CPET. Exercise programs used were the modified Bruce grading program. The termination criteria for exercise test was according to the ACMS (15): i) Patient requirements; ii) with the raise of exercise load, SBP has decreased $10 \mathrm{mmHg}$ comparing to baseline, accompanied with other ischemic evidence; iii) the occurrence of moderate to severe angina; iv) nervous system symptoms such as ataxia, dizziness and close to syncope; v) signs of hypoperfusion: cyanosis, pale; vi) serious arrhythmias: ventricular tachycardia, ventricular flutter and ventricular fibrillation; vii) ST-segment of severe depression in oblique type or horizontal type depressed $\geq 2 \mathrm{~mm}$ or ST-segment elevation $\geq \mathrm{Tmm}$; and viii) monitoring blood pressure difficult or not reaching the target heart rate.

Before exercise, we measured and recorded blood pressure, HRrest (bmp), resting ECG. In the test, blood pressure, ECG, heart rate at the end of each grade, HRpeak and peak $\mathrm{VO}_{2} / \mathrm{kg}(\mathrm{ml} / \mathrm{kg} / \mathrm{min})$ were recorded as required. The ECG was recorded until 6 min after exercise or ST-segment returned to resting state by the end. The following formulas were used to calculate rhR, HRRes, $\mathrm{HRR}_{1-6}$ :

$$
\mathrm{rHR}=(\text { HRpeak }) /(220-\text { age }) \times 100 \%
$$

\section{HRRes $=($ HRpeak - HRrest $) /(220-$ age - HRrest $) \times 100 \%$}

The patients continued to walk slowly after the exercise test was completed. While $\mathrm{HRR}_{1-6}$ refers to an absolute difference between heart rate reaching peak during exercise and heart rate at 1,2,3, 4, 5 and 6 min after movement cessation, namely HRR value at each time-point by the end of exercise test.

Individual exercise prescription was formulated. According to Chinese Guidelines of Exercise Therapy in Diabetes Mellitus (2) and exercise test and exercise prescription guidelines by American College of Sports Medicine to formulate individual exercise prescription. The general principle of individual exercise prescription formulation is as follows: (needs to be adjusted according to the individual situation). i) Exercise mode: Aerobic exercise: Brisk walking, bicycle; resistance movement: Elastic band resistance training, fluid resistance movement; flexibility: Stretching of muscles or ligaments; balance movement: Single foot standing, throwing and catching a ball. ii) Movement time: Exercise time 30-60 min, the duration of the effective heart rate reaching at least 20-30 min, not including the warm-up before exercise and finishing after exercise. iii) Exercise intensity: Heart rate reserve method. 
Table I. Changes of body parameters and biochemical indexes before and after treatment.

\begin{tabular}{lrr}
\hline Index & Before & \multicolumn{1}{c}{ After } \\
\hline BMI $\left(\mathrm{kg} / \mathrm{m}^{2}\right)$ & $25.55 \pm 3.17$ & $24.56 \pm 2.37^{\mathrm{b}}$ \\
Abdominal girth $(\mathrm{cm})$ & $93.10 \pm 9.74$ & $91.65 \pm 9.23^{\mathrm{b}}$ \\
FPG $(\mathrm{mmol} / \mathrm{l})$ & $9.41 \pm 4.11$ & $8.06 \pm 2.15^{\mathrm{a}}$ \\
HbAlc $(\%)$ & $8.25 \pm 1.78$ & $7.45 \pm 1.07^{\mathrm{a}}$ \\
\hline
\end{tabular}

Compared with pre-treatment, ${ }^{\mathrm{a}} \mathrm{P}<0.05,{ }^{\mathrm{b}} \mathrm{P}<0.01$.

Table II. Comparison of $\mathrm{CR}, \mathrm{HRR}_{1-6}$ before and after treatment.

\begin{tabular}{lcc}
\hline Index & Before & After \\
\hline HRrest (bmp) & $74.37 \pm 8.40$ & $69.77 \pm 5.91^{\mathrm{b}}$ \\
HRpeak (bmp) & $129.23 \pm 16.32$ & $140.50 \pm 13.41^{\mathrm{b}}$ \\
rHR & $0.81 \pm 0.11$ & $0.88 \pm 0.07^{\mathrm{b}}$ \\
HRRes & $0.65 \pm 0.18$ & $0.77 \pm 0.13^{\mathrm{b}}$ \\
$\mathrm{HRR}_{1}$ (bmp) & $17.17 \pm 7.98$ & $20.47 \pm 9.01^{\mathrm{a}}$ \\
$\mathrm{HRR}_{2}$ (bmp) & $27.33 \pm 10.18$ & $34.20 \pm 10.62^{\mathrm{a}}$ \\
$\mathrm{HRR}_{3}$ (bmp) & $32.67 \pm 9.86$ & $39.93 \pm 11.49^{\mathrm{a}}$ \\
$\mathrm{HRR}_{4}$ (bmp) & $40.03 \pm 12.96$ & $46.43 \pm 14.26^{\mathrm{a}}$ \\
$\mathrm{HRR}_{5}$ (bmp) & $42.90 \pm 13.18$ & $49.93 \pm 13.58^{\mathrm{a}}$ \\
$\mathrm{HRR}_{6}$ (bmp) & $43.57 \pm 12.01$ & $51.10 \pm 13.05^{\mathrm{a}}$ \\
\hline
\end{tabular}

Compared with pre-treatment, ${ }^{\mathrm{a}} \mathrm{P}<0.05$, ${ }^{\mathrm{b}} \mathrm{P}<0.01$. CR, chronotropic response; HRpeak, peak heart rate; rHR, heart rate value; HRRes, heart rate reserve rate; HRR, heart rate recovery.

Combining with perceived exertion (RPE) scale, peak $\mathrm{VO}_{2} / \mathrm{kg}$, that is, to achieve the RPE 13-14 level or the selected exercise according to the intensity of $50-60 \%$ peak $\mathrm{VO}_{2} / \mathrm{kg}$. Target heart rate $=($ HRpeak - HRrest $) \mathrm{x}$ intensity $\%+$ HRrest. iv $)$ Exercise frequency: 3-7 times/week. If the amount of exercise each time is greater, it can be spaced over one or two days, if the amount of each exercise is less and the body allows, then adhere to 7 times/week.

Implementation of exercise therapy. According to the individual situation of patients to decide to wear telemetric ECG monitor or finger pulse oximeter and to comply with exercise prescriptions. The rating of RPE has reached 13-14 grades in the process of movement. Before and after exercise test, blood glucose, blood pressure, and heart rate were determined and attention was given to observe ECG, blood pressure response and discomfort during movement.

To complete repeated symptom-limited CEPT, calculation and statistics of indexes. The implementation methods of symptom-limited CPET and the calculation of each index were the same as above. The relevant physical parameters and biochemical indicators were arranged as statistical information.

Statistical analysis. Data were entered into the database, using SPSS statistical analysis software (SPSS, Inc., Chicago, IL,
Table III. Comparison of exercise capacity before and after treatment.

\begin{tabular}{lcc}
\hline Index & Before & After \\
\hline Exercise time $(\mathrm{sec})$ & $703.10 \pm 196.47$ & $873.80 \pm 177.79^{\mathrm{a}}$ \\
Peak $\mathrm{VO}_{2} / \mathrm{kg}(\mathrm{ml} / \mathrm{kg} / \mathrm{min})$ & $16.99 \pm 3.96$ & $21.40 \pm 4.94^{\mathrm{a}}$ \\
Peak $\mathrm{VO}_{2} / \mathrm{kg}$ pred $(\%)$ & $70.97 \pm 11.82$ & $89.17 \pm 13.68^{\mathrm{a}}$ \\
\hline
\end{tabular}

Compared with pre-treatment, ${ }^{\mathrm{a}} \mathrm{P}<0.01$.

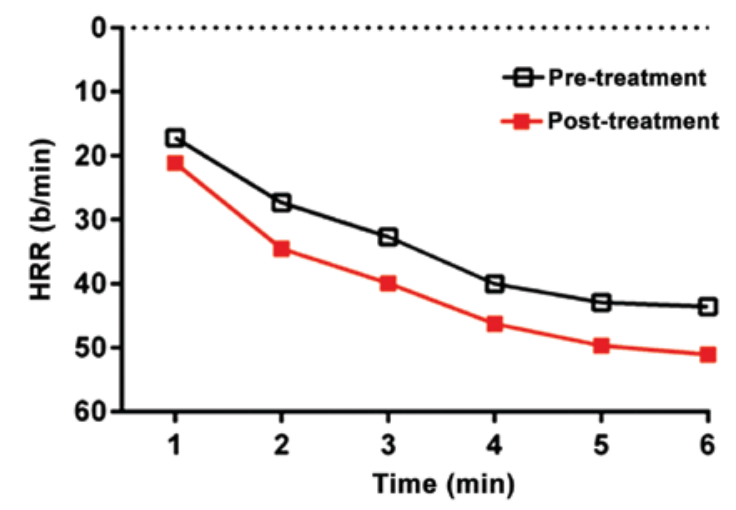

Figure 1. HRR curve diagram before and after treatment. HRR, heart rate recovery.

USA). Quantitative data were expressed as mean $\pm \mathrm{SD}$, the income data were under normality and homogeneity of variance test. According to the data characteristics, in line with the conditions or data conversion according with the conditions, before and after the treatment itself compared using paired samples t-test. Pearson correlation analysis was used for correlation analysis, if not meeting the conditions the non-parametric test was selected, correlation analysis was used by Spearman correlation analysis. $\alpha=0.05$ was regarded as test level, $P<0.05$ was considered to indicate a statistically significant difference.

\section{Results}

Comparison of body parameters and biochemical indexes before and after treatment. After treatment, BMI, abdominal girth, FPG and HbAlc were lower than pre-treatment. The difference was statistically significant $(\mathrm{P}<0.01)$ (Table I).

Comparison of CR and HRR index before and after treatment. Following treatment, HRrest was decreased significantly, whereas HRpeak, rHR, HRRes and HRR ${ }_{1-6}$ increased significantly $(\mathrm{P}<0.05)$ (Table II). The exercise test was completed before and after treatment, and the 1-6 min HRR curve diagram is shown in Fig. 1.

Comparison and correlation analysis of exercise capacity indexes before and after treatment. After treatment, exercise time, peak $\mathrm{VO}_{2} / \mathrm{kg}$ and peak $\mathrm{VO}_{2} / \mathrm{kg}$ were significantly higher than pre-treatment $(\mathrm{P}<0.01)$ (Table III). Pearson correlation analysis showed that Peak $\mathrm{VO}_{2} / \mathrm{kg}$ was 


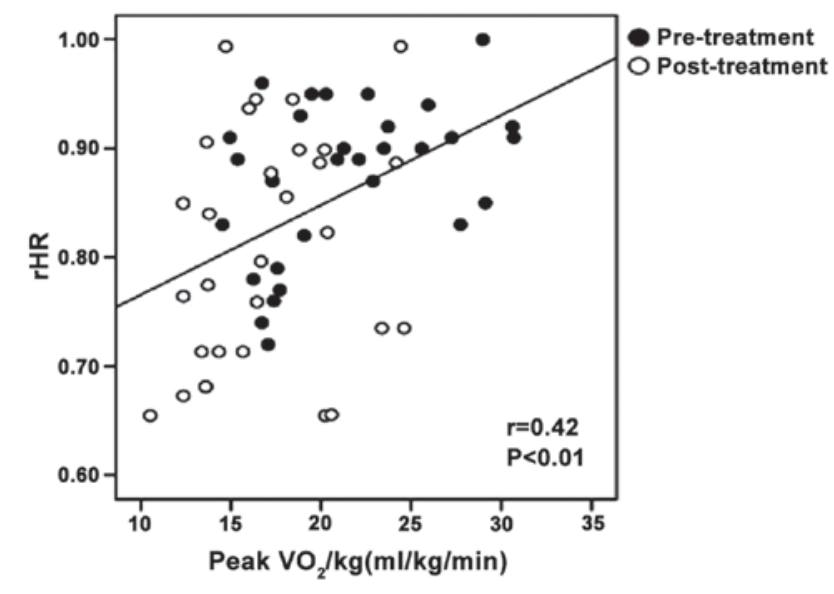

Figure 2. Peak $\mathrm{VO}_{2} / \mathrm{kg}$ and rHR-related scatter diagram. rHR, heart rate value.

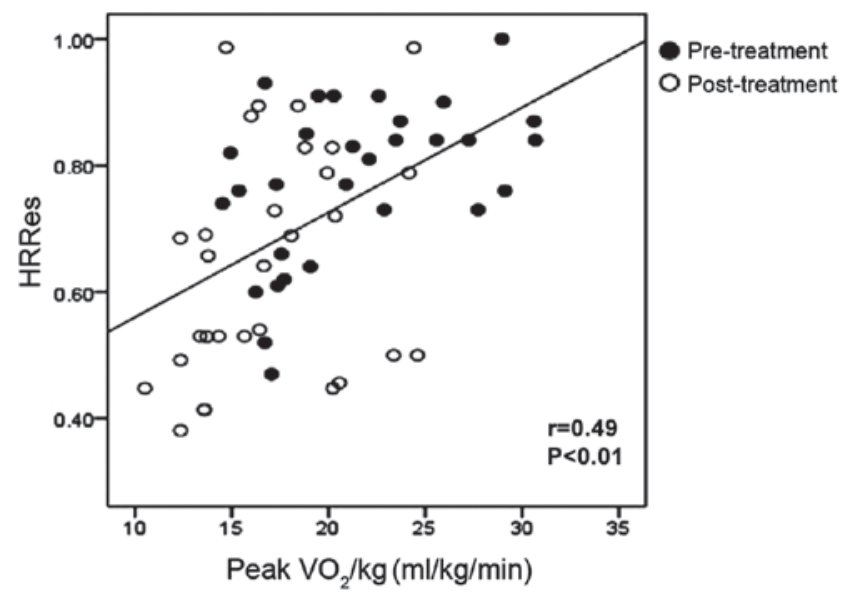

Figure 3. Peak $\mathrm{VO}_{2} / \mathrm{kg}$ and HRRes-related scatter diagram. HRRes, heart rate reserve rate.

positively correlated with rHR, HRRes (r-value: 0.42 and 0.49 , both $\mathrm{P}<0.01$ ) (Figs. 2 and 3).

\section{Discussion}

The heart rate at any time-point responses affects the dynamic balance of distribution of the sympathetic nerve and vagal tone in the autonomic nervous system. Under resting conditions, heart rate receives double regulation of sympathetic nerve and vagus nerve, predominantly vagus nerve (3). The sympathetic tone increased and/or vagal tone decreased causing the heart rate to be adjusted to the normal heart rate with a decrease of physical activity, which showed the increase of HRrest. At the initial stage of exercise, the vagus nerve was inhibited and the heart rate increased rapidly. Sympathetic nerve excitability reached a peak in a few seconds. After that, the heart rate increased slowly with the increase of the concentration of catecholamine in the blood. The heart rate during exercise could not be increased with the demands of the body metabolism, or could not maintain stability in the performance of CI (16). At the completion of exercise with sympathetic nerve tension retreat and vagal activation, a rapid decrease of heart rate, especially in the $30 \mathrm{sec}$ after termination was induced (17). HRR of healthy adults after exercise can be classified as the following three stages (18): i) Fast recovery period with short duration, the activity of vagus nerve predominated just at the end of movement; ii) then entered the slow recovery phase with longer duration, performance for the sympathetic nerve activity recovered gradually; and iii) accessed to the stable period with slight fluctuation in the end, the sympathetic nervous tension and the tension of the vagus nerve are in a relatively balanced state. The HRR will slow down also the time will prolong if regulation is impaired.

It has been found that the CI index in T2DM patients with no metabolic syndrome was significantly lower than that of patients with metabolic syndrome (19). In addition, autonomic nerve dysfunction dominated by the sympathetic and vagal nerve exists in T2DM patients with no cardiovascular disease $(20,21)$. A study of 1,341 T2DM patients showed that $35.7 \%$ with CI during the exercise test and long-term follow-up showed that it was closely related to the malignant endpoint events (22). In another study, 2,123 men (mean age, 47 46 years) underwent a complete health examination, and all subjects in the first examination were excluded from coronary heart disease and T2DM. After 5 years, the subjects underwent a secondary check, which revealed 137 (4.4\%) subjects suffering from T2DM (23). The results suggested that CI was associated with the incidence of T2DM during test in a healthy male population, and was independent of other risk factors.

The delay of HRR is closely related to the incidence of DM (24). A study on a group of male T2DM patients with a follow-up lasting 14.9 years showed that the HRR delay is an independent predictor of cardiovascular disease and the occurrence of all-cause of death $(24,25)$. HRR has a certain correlation with myocardial ischemia in patients with T2DM (26), showing that abnormal HRR may decrease the exercise capacity of patients in exercise test and it is related to the increase of all-cause mortality and cardiovascular events risk (27).

For the selected 30 patients of T2DM before exercise therapy, rHR (0.81 \pm 0.11$)$, HRRes $(0.65 \pm 0.18)$, $\mathrm{HRR}_{1}(17.17 \pm 7.98 \mathrm{bmp})$, and $\mathrm{HRR}_{2}(27.33 \pm 10.18 \mathrm{bmp})$ were significantly lower than normal. The results are in agreement with data from other reports showing abnormal CI, and HRR exist widely in T2DM patients $(25,26)$. Therefore, the treatment of CI, abnormal HRR and other related manifestations which were caused by autonomic nerve dysfunction in patients with T2DM is imminent.

By selecting coronary heart disease, hypertension, T2D patients as the subjects, some studies have found short-term individualized rehabilitation exercise on cardiac autonomic nerve dysfunction with immediate effect and improvement of parasympathetic nerve function was better than that of sympathetic nerve function in exercise (28). In a previous study, 4,503 T2DM patients were randomly divided into the exercise and control groups, with the former being offered lifestyle intervention and training, and the latter being given conventional treatment and education (29). One year later, the results showed that the exercise group lost weight, HRrest decreased, HRRes increased, with an acceleration in HRR and the reduction in weight and exercise capacity upgrade were independent influencing factors for HRR improvement. By contrast, in the 
control group, these indexes had no obvious change, thus HRR can be an important indicator in assessing cardiovascular risk (29). Our study found that after 12 weeks of exercise therapy, HRrest $(74.37 \pm 8.40$ vs. $69.77 \pm 5.91$ bmp) decreased, HRpeak (129.23 \pm 16.32 vs. $140.50 \pm 13.41 \mathrm{bmp})$, rHR $(0.81 \pm 0.11$ vs. $0.88 \pm 0.07)$ and HRRes $(0.65 \pm 0.18$ vs. $0.77 \pm 0.13)$ increased, and HRR $\left(\mathrm{HRR}_{1}: 17.17 \pm 7.98\right.$ vs. $20.47 \pm 9.01 \mathrm{bmp}$, $\mathrm{HRR}_{2}: 27.33 \pm 10.18$ vs. $34.20 \pm 10.62 \mathrm{bmp}$ ) were significantly higher than before. Our results also showed that exercise can improve cardiac autonomic nerve balance and regulation in T2DM patients. The vagus nerve retreat in exercise early stage and full activation of the sympathetic nerve activity in the later period of exercise leads to an increase in heart rate. Additionally, sympathetic rapid retreat resulted in a decrease in heart rate and served as a protective mechanism for the heart and a fulfillment for the body's metabolic demand.

T2DM is considered to be a disease of the lack of exercise (body inertia) (30), with more than $80 \%$ of T2DM being related to obesity and body inertia. The study found that low exercise tolerance is an independent risk factor for mortality in patients with T2DM (31). Since the 1960s, with the development of rehabilitation medicine and the performance of rehabilitation training for cardiovascular disease, exercise prescription began to be valued. The Chinese Guideline for Exercise in Diabetes (12) recommended that the main content of the exercise prescription includes exercise program, exercise intensity, exercise timing, exercise duration and exercise frequency. In addition, the exercise intensity is the core of exercise prescription and each time of training should consists of three parts, including preparation, basic activities and finishing part.

Exercise could not only improve the risk factors of diabetes attacks, glucose tolerance, fasting glucose damaged state, prevent the occurrence of complications, the same can enhance exercise capacity in patients with T2DM, improve complication process and prognosis (12). The exercise capacity directly reflects the level of individual health status and self-care ability. Our investigation found that after 12 weeks of exercise training, with exercise test time was prolonged, peak $\mathrm{VO}_{2} / \mathrm{kg}$ and peak $\mathrm{VO}_{2} / \mathrm{kg}$ pred increased, suggesting that the exercise capacity of patients may be enhanced. Previous findings have shown that peak $\mathrm{VO}_{2} / \mathrm{kg}$ in patients with T2DM increased after exercise training may due to strengthening of the major muscle groups caused by addition in the uptake of oxygen, enhancement of heart diastolic function, increase of left ventricular mass and the increase of muscle fiber and capillary density (32).

It was demonstrated that the improvement of cardiac autonomic nerve function in patients with T2DM was partly due to the increase of exercise tolerance (33). The vagal activity was positively correlated with exercise tolerance in healthy people and patients with chronic diseases (34). Our investigation also demonstrated that peak $\mathrm{VO}_{2} / \mathrm{kg}$ was positively correlated with HRRes and rHR, showing that enhancement of exercise capacity was closely related to the improvement of autonomic nerve function.

Exercise training has been shown to increase insulin receptor sensitivity, improve lipid metabolism, decrease blood sugar and reduce weight of T2DM patients (12). In the present study, BMI decreased, abdominal girth was reduced, as were FPG and HbAlc, as suggested by previous studies $(35,36)$.
The present study has demonstrated that exercise training improved the CI and HRR delay in T2DM patients and promote the adaptability of the cardiovascular system to exercise stress, by regulating the function of cardiac autonomic nerves. In addition, the training also improved the exercise capacity and optimized the body metabolism index. Exercise therapy plays an important role in the treatment of DM as one of 'five carriages'. This study is conducive to the promotion of exercise rehabilitation effect and health education for the patients, which could improve the exercise compliance.

The deficiency of this study was that the limitation of clinical sources led to a low number of patients being enrolled in the study. In future studies, we intend to continue to expand the sample size for further study and analysis.

\section{References}

1. Yang W, Lu J, Weng J, Jia W, Ji L, Xiao J, Shan Z, Liu J, Tian H, Ji Q, et al; China National Diabetes and Metabolic Disorders Study Group: Prevalence of diabetes among men and women in China. N Engl J Med 362: 1090-1101, 2010.

2. Chinese Diabetes Society (CDS): Chinese guidelines of exercise therapy in diabetes mellitus. Chinese Diabetes Society, Beijing, pp46-57, 2013.

3. Sigal RJ, Kenny GP, Wasserman DH, Castaneda-Sceppa C and White RD: Physical activity/exercise and type 2 diabetes: a consensus statement from the American Diabetes Association. Diabetes Care 29: 1433-1438, 2006.

4. Brubaker PH and Kitzman DW: Chronotropic incompetence: causes, consequences, and management. Circulation 123: 1010-1020, 2011.

5. Dresing TJ, Blackstone EH, Pashkow FJ, Snader CE, Marwick TH and Lauer MS: Usefulness of impaired chronotropic response to exercise as a predictor of mortality, independent of the severity of coronary artery disease. Am J Cardiol 86: 602-609, 2000.

6. Wang ST: Effect of aerobic exercise on the balance of cardiovascular autonomic nerve. Beijing Sport University, pp6-9, 2006.

7. Young LH, Wackers FJ, Chyun DA, Davey JA, Barrett EJ, Taillefer R, Heller GV, Iskandrian AE, Wittlin SD, Filipchuk N, et al; DIAD Investigators: Cardiac outcomes after screening for asymptomatic coronary artery disease in patients with type 2 diabetes: the DIAD study: a randomized controlled trial. JAMA 301: 1547-1555, 2009.

8. Kaplan JM, Okin PM and Kligfield P: The diagnostic value of heart rate during exercise electrocardiography. J Cardiopulm Rehabil 25: 127-134, 2005.

9. Lin XF, Liu Z and Dong FY: Application and research progress of heart rate recovery in the rehabilitation of cardiovascular diseases. Chinese J Physical Med Rehabil 35: 498-500, 2013 (In Chinese).

10. Halon DA, Dobrecky-Mery I, Gaspar T, Azencot M, Yaniv N, Peled N and Lewis BS: Heart rate recovery after exercise and coronary atheroma in asymptomatic individuals with type 2 diabetes mellitus: a study using 64-slice coronary CT angiog paphy. Int J Cardiol 145: 102-103, 2010.

11. Chacko KM, Bauer TA, Dale RA, Dixon JA, Schrier RW and Estacio RO: Heart rate recovery predicts mortality and cardiovascular events in patients with type 2 diabetes. Med Sci Sports Exerc 40: 288-295, 2008.

12. Zhu L, Liu YN and Yu RJ: Clinical pulmonary function. 1st edition. People's Health Publishing House, Beijing, pp296-297, 2004

13. Colberg SR, Sigal RJ, Fernhall B, Regensteiner JG, Blissmer BJ, Rubin RR, Chasan-Taber L, Albright AL and Braun B; American College of Sports Medicine; American Diabetes Association: Exercise and type 2 diabetes: the American College of Sports Medicine and the American Diabetes Association: joint position statement. Diabetes Care 33: e147-e167, 2010.

14. American Diabetes Association: Standards of medical care in diabetes - 2010. Diabetes Care 33 (Suppl 1): S11-S61, 2010.

15. American College of Sports Medicine (ACSM): ACSM's Guidelines for Exercise Testing and Prescription. 6th edition. Lippincott Williams \& Wilkins, Philadelphia, PA, 2000. 
16. Orso F, Baldasseroni S and Maggioni AP: Heart rate in coronary syndromes and heart failure. Prog Cardiovasc Dis 52: 38-45, 2009.

17. Lauer MS: Autonomic function and prognosis. Cleve Clin J Med 76 (Suppl 2): S18-S22, 2009.

18. Goldberger JJ, Le FK, Lahiri M, Kannankeril PJ, Ng J and Kadish AH: Assessment of parasympathetic reactivation after exercise. Am J Physiol Heart Circ Physiol 290: H2446-H2452, 2006.

19. Gao M, Chen W, Gong ZK, Han L and Zhang L: Correlation between chronotropic incompetence and metabolic equivalents in patients with type 2 diabetes mellitus and concomitant metabolic syndrome. Panminerva Med 57: 115-119, 2015.

20. Ho JS, Fitzgerald SJ, Barlow CE, Cannaday JJ, Kohl HW III Haskell WL and Cooper KH: Risk of mortality increases with increasing number of abnormal non-ST parameters recorded during exercise testing. Eur J Cardiovasc Prev Rehabil 17: 462-468, 2010

21. Zhou J, Gao M and Chen W: Relationship between heart rate recovery after exercises and mobility in type 2 diabetes patients. Acta Acad Med Xuzhou 35: 238-241, 2015.

22. Ho PM, Maddox TM, Ross C, Rumsfeld JS and Magid DJ: Impaired chronotropic response to exercise stress testing in patients with diabetes predicts future cardiovascular events. Diabetes Care 31: 1531-1533, 2008.

23. Jae SY, Kurl S, Laukkanen JA, Heffernan KS, Choi YH and Park WH: Chronotropic response to exercise and risk of type 2 diabetes in men. Eur Heart J 34: 5815, 2013.

24. Jae SY, Carnethon MR, Heffernan KS, Fernhall B, Lee MK and Park WH: Heart rate recovery after exercise and incidence of type 2 diabetes in men. Clin Auton Res 19: 189-192, 2009.

25. Cheng YJ, Lauer MS, Earnest CP, Church TS, Kampert JB, Gibbons LW and Blair SN: Heart rate recovery following maximal exercise testing as a predictor of cardiovascular disease and all-cause mortality in men with diabetes. Diabetes Care 26 2052-2057, 2003.

26. Georgoulias P, Demakopoulos N, Orfanakis A, Xydis K Xaplanteris P, Vardas P and Fezoulidis I: Evaluation of abnormal heart-rate recovery after exercise testing in patients with diabetes mellitus: correlation with myocardial SPECT and chronotropic parameters. Nucl Med Commun 28: 165-171, 2007.
27. Georgoulias P, Demakopoulos N, Valotassiou V, Orfanakis A, Zaganides A, Tsougos I and Fezoulidis I: Long-term prognostic value of heart-rate recovery after treadmill testing in patients with diabetes mellitus. Int J Cardiol 134: 67-74, 2009.

28. Li J, Chen W and Gao M: Effect of individualized exercise on cardiac autonomic nerve function. J Chinese Modern Med 16: 27-31, 2014 (In Chinese).

29. Ribisl PM, Gaussoin SA, Lang W, Bahnson J, Connelly SA, Horton ES, Jakicic JM, Killean T, Kitzman DW, Knowler WC, et al; Look AHEAD Research Group: Lifestyle intervention improves heart rate recovery from exercise in adults with type 2 diabetes: results from the Look AHEAD study. J Obes 2012: 309196, 2012.

30. Schwarz PE, Lindström J, Kissimova-Scarbeck K, Szybinski Z, Barengo NC, Peltonen M and Tuomilehto J; DE-PLAN project: The European perspective of type 2 diabetes prevention: diabetes in Europe - prevention using lifestyle, physical activity and nutritional intervention (DE-PLAN) project. Exp Clin Endocrinol Diabetes 116: 167-172, 2008.

31. Church TS, LaMonte MJ, Barlow CE and Blair SN: Cardiorespiratory fitness and body mass index as predictors of cardiovascular disease mortality among men with diabetes. Arch Intern Med 165: 2114-2120, 2005.

32. Albright A, Franz M, Hornsby G, Kriska A, Marrero D, Ullrich I and Verity LS: American College of Sports Medicine position stand. Exercise and type 2 diabetes. Med Sci Sports Exerc 32: 1345-1360, 2000.

33. Pagkalos M, Koutlianos N, Kouidi E, Pagkalos E, Mandroukas K and Deligiannis A: Heart rate variability modifications following exercise training in type 2 diabetic patients with definite cardiac autonomic neuropathy. Br J Sports Med 42: 47-54, 2008.

34. Hedelin R, Bjerle P and Henriksson-Larsén K: Heart rate variability in athletes: relationship with central and peripheral performance. Med Sci Sports Exerc 33: 1394-1398, 2001.

35. Reitman JS, Vasquez B, Klimes I and Nagulesparan M: Improvement of glucose homeostasis after exercise training in non-insulin-dependent diabetes. Diabetes Care 7: 434-441, 1984.

36. Kraus WE and Levine BD: Exercise training for diabetes: The 'strength' of the evidence. Ann Intern Med 147: 423-424, 2007. 